\title{
Medial Clavicle Fracture Dislocation Surgically Treated: Case Report
}

\section{Miguel Frias", Renato Ramos, Marco Bernardes, Tiago Pinheiro Torres and Pedro Lourenço}

Centro Hospitalar de Vila Nova de Gaia/Espinho, Portugal

*Corresponding author: Miguel Frias, Centro Hospitalar de Vila Nova de Gaia/Espinho, Porto, Portugal

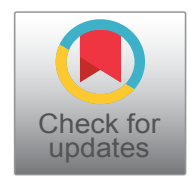

\begin{abstract}
The medial clavicle fracture is a rare injury and almost all the times conservatively treated. Therefore, the number of operations reported in the literature are limited. Posterior dislocation can result in serious complications and such injuries require rapid diagnosis followed by effective treatment to avoid future complications. The present case reports a case of a 33-year-old healthy man that was involved in a high-fall, being diagnosed with a rare displaced medial clavicular fracture associated with an unstable posterior lateral fragment dislocation surgically treated. Imagiological images and intraoperative photographs are presented. The authors collected this case in order to report this rare and complex injury. The treatment chosen to manage seems to give a good functional and radiographic recovery.
\end{abstract}

\section{Introduction}

The medial clavicle fracture is a rare injury, accounting for only $2 \%$ to $3 \%$ of all clavicle fractures [1]. The majority of medial clavicle fractures can be successful conservatively treated with success [2]. Because of the rarity of medial clavicle fractures, the number of operations reported in the literature is limited $[3,4]$.

In polytrauma patients, a clavicle fracture is easily diagnosed during a primary survey and may indicate underlying thoracic injury, as the rate and extent of the concomitant thoracic injury are high [5]. Two types of medial fracture dislocation of the clavicle are described -anterior and posterior- according to the direction of the displacement of the fractured clavicle in relation to the sternum [6]. Posterior dislocation can result in serious complications, including compression or tearing of adjacent neural and vascular structures, injury to the trachea and esophagus and the possibility of pneumothorax [7].

Although rare, such injuries require rapid diagnosis followed by effective treatment to avoid future complications [8]. Specifically, periarticular and intraarticular fractures remain a therapeutic challenge: The medial fragment is too small to stabilize with common linear implants, and bending forces that occur during shoulder motion are disproportionately high [9]. Indications for surgery have traditionally included open fractures, soft-tissue damage, or neurovascular impairment [9].

Although not every fracture-dislocation of the medial clavicle needs a surgical intervention, the treatment should occur in a level one trauma center because of the potentially life-threatening effects and co-injuries and the fact that there is a kind of reluctance for orthopedic surgeons to operate in this usually unfamiliar area [10].

The present case reports a rare displaced medial clavicular fracture associated with an unstable posterior lateral fragment dislocation and its management.

\section{Case Report}

A 33-year-old healthy man was involved in a highfall during his work, being presented to the nearest emergency department. The initial evaluation was performed according to the ATLS protocol. His complaints were shoulder and neck pain. No open injuries, dyspnea, dysphagia, or numbness were reported, he presented hemodynamically stable. X-rays and CT-scan were performed and revealed a right posterior displaced medial clavicle fracture, close to but 

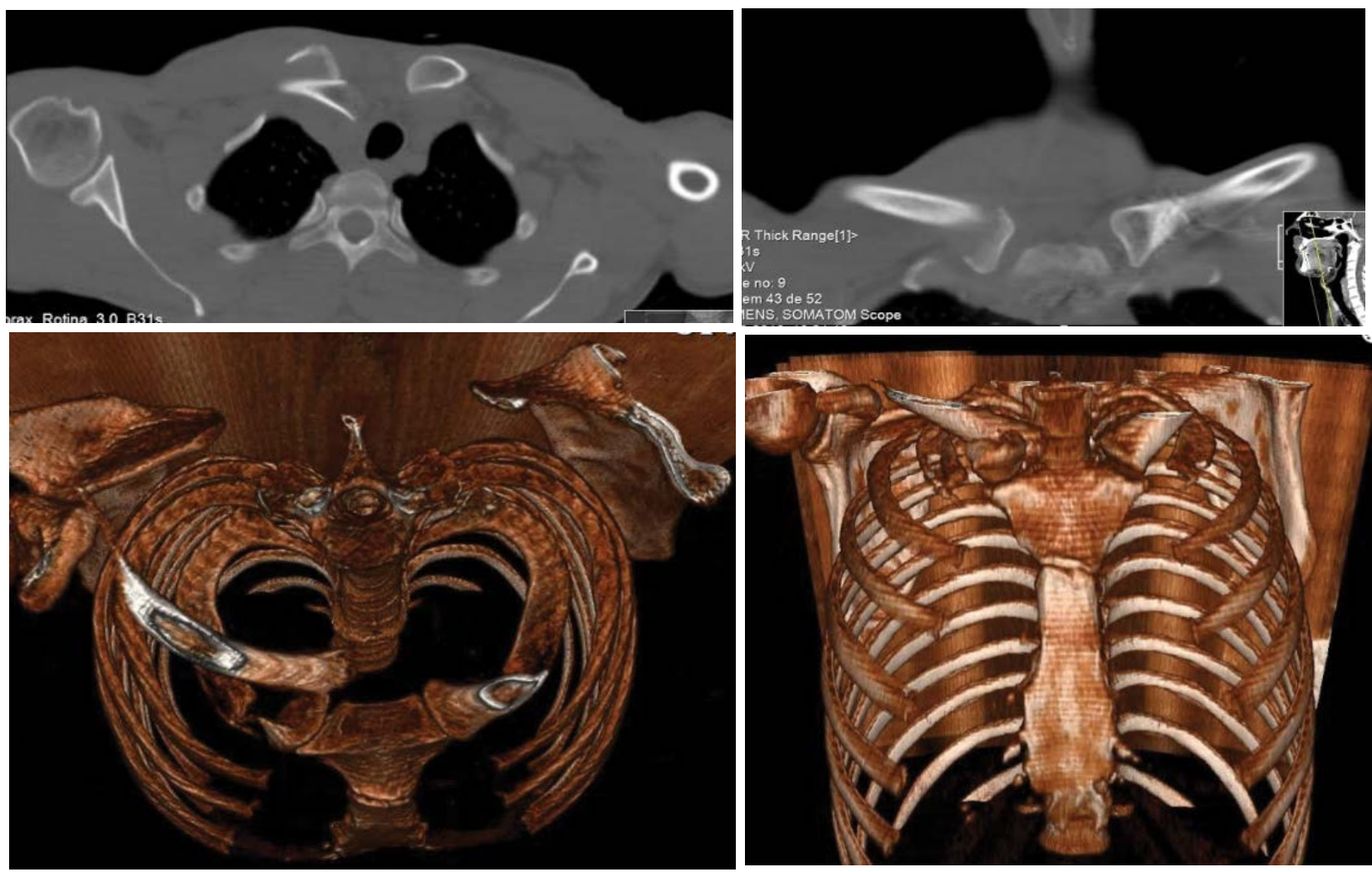

Figure 1: Pre-op 2D and CT- scan 3D reconstruction; Axial and coronal views.

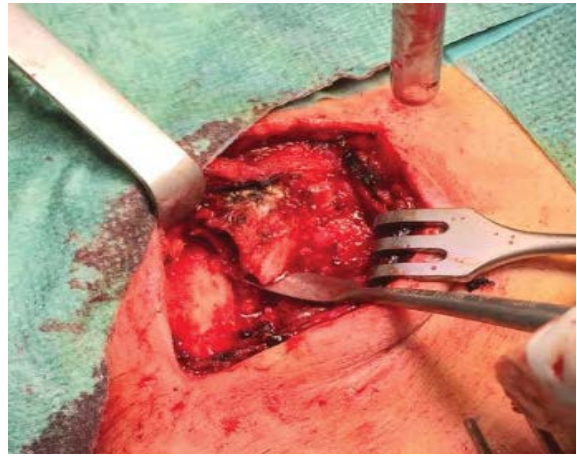

A

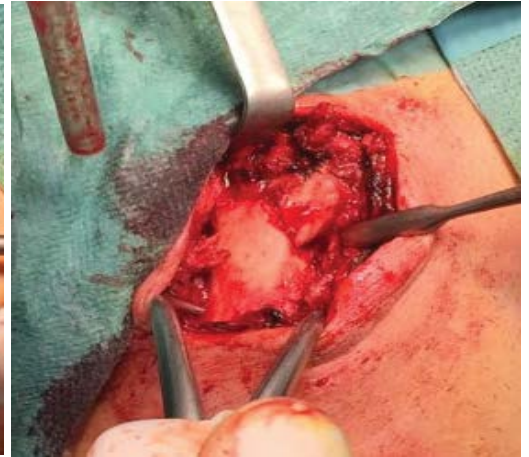

B

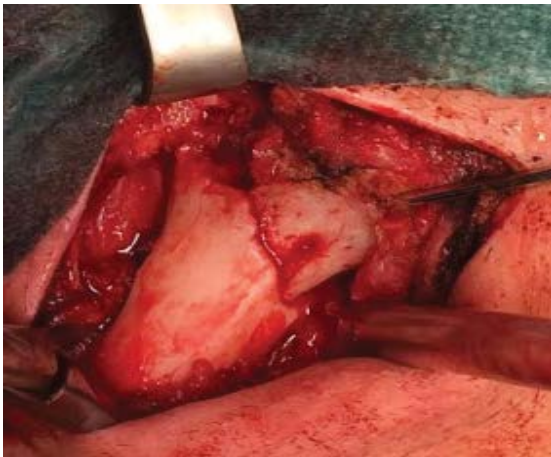

C

Figure 2: A) Fracture pattern pre-attempting open reduction; B) Reduction of the fracture, instrument-assisted; C) Reduced fracture.

with no compression of the underlying neurovascular or gastro or airway structures (See Figures 1). Due to these findings, it was determined that he would require surgery for an open reduction an internal fixation of the right medial clavicle fracture, for which appropriate consent was obtained.

The patient was placed in the beach chair position under general anesthesia and antibiotic prophylaxis was administered. Closed reduction was unsuccessfully attempted. An oblique oriented incision was centered over the clavicular fracture site and subsequently extended to the right sternoclavicular joint. The underlying clavicle was exposed, and minimal soft tissue dissection was bluntly performed with a periosteal elevator. Care was taken to preserve softtissue attachments and bone fragments. The clavicular fracture was identified and posterior dislocation of the lateral fragment was confirmed and no intra-articular sternoclavicular extension was observed. Debridement was performed and the reduction was successfully attempted, avoiding and protecting the neurovascular structures (See Figure 2A, Figure 2B and Figure 2C). Internal fixation with an anterior lateral locking LCDCP clavicle plate with locking and non-locking screws (VARIAX STRYKER ${ }^{\circledR}$ ) was performed.

Two cerclages sutures with Ethibond ${ }^{\circledR}$ around the plate and fracture were performed to reinforce the construct. Adequate stability and hemostasis were achieved, and the wound was closed in layers. The reduction and stability of the fracture were confirmed at the end of the procedure with X-ray image intensifier (See Figure 3A and Figure 3B). 


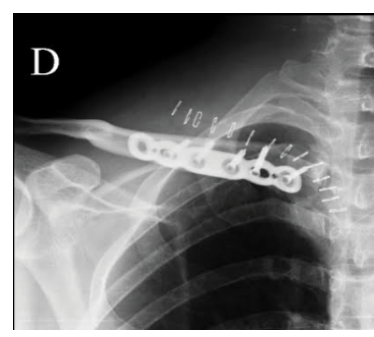

A

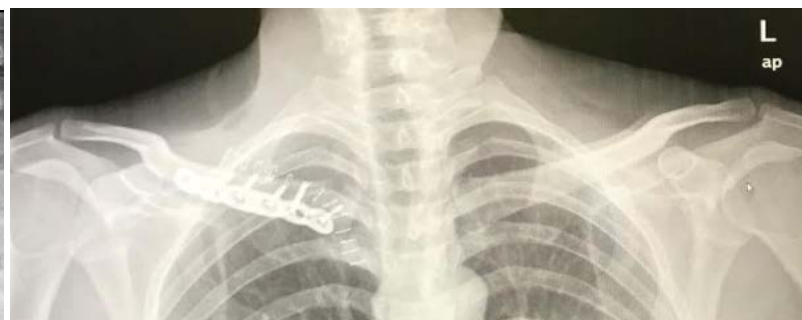

B

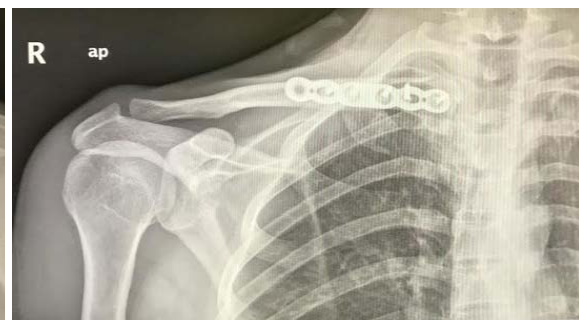

C

Figure 3: A and B) Immediate postop X-rays; C) 12 weeks control X-ray.

The patient was immobilized with a sling reinforced with a thoracic band. Passive wrist and elbow range of motion and pendulum exercises of the shoulder were allowed. Staples were retrieved fifteen days after surgery. Follow-up was performed on the $2^{\text {nd }}$ week, $4^{\text {th }}$ week, $3^{\text {rd }}$ and $6^{\text {th }}$ months after surgery. Passive range of motion of the shoulder was allowed after the first month, and active motion exercises were progressively introduced. Six months after surgery the control X-ray showed a completely united fracture (See Figure 3C). No functional pain or local tenderness at the fracture site was noticed and almost full range of motion of the right shoulder was restored on the latest follow-up. The Disabilities of the Arm, Shoulder, and Hand (DASH) score was performed at final follow-up with a value of 11.7. No major or minor complications were registered.

\section{Discussion}

Surgical treatment of medial clavicle fractures is indicated when a closed reduction is unsuccessful or when the injury progresses to recurrent instability and pain, resulting in great discomfort and disability; however, an optimal, standardized operative treatment has not yet been established.

Most studies have reported poorer outcomes when displaced medial clavicle fractures are non-surgically treated. In a prospective study, Nowak, et al. in 222 diagnosed clavicle fractures, only 4 were medial end clavicle fractures, in which 2 of them were painful at the latest follow-up, with one nonunion reported [11]. Throckmorton, et al. reviewed 57 medial end clavicle fractures retrospectively, in which only four patients with open fractures were surgically treated. On the patients in which the CT-scan was performed $44 \%$ of medial end fractures were minimally displaced $(<2 \mathrm{~mm}), 23 \%$ had $2-10 \mathrm{~mm}$, and $33 \%$ had $>10 \mathrm{~mm}$ of displacement. After a mean of 16 months, the majority of patients still reported mild to severe pain [12]. Regarding the clinical case reported, the CT-scan had a primordial importance on the evaluation of neurovascular and airway concomitant injuries, on the evaluation of fracture type and displacement, and surgical planning.

Keisuke, et al. in a retrospective study, analyzed 10 patients diagnosed with medial periarticular dislocated fractures surgically treated, in all cases result of a highenergy trauma. Mean age was 34-years-old, and pre- operative CT-scan was obtained in 6 patients, with multiple injuries. Half of the patients were operated within 10 days after injury [9]. The authors used locking plates, in 6 of 10 patients, three screws were placed in the medial fragment or the sternum. Nine of ten fractures united clinically and radiographically with an almost free range of motion and minimal impairment at the latest follow-up. In one patient, the authors observed plate loosening and superficial wound infection and had to remove the osteosynthesis material and perform a surgical site debridement. In seven of nine patients with fracture union, the plate was removed after a mean of 12 months [9]. The authors also recommended a CT-scan for patients who are considered for operative treatment, for surgical planning [9].

Frima, et al. in a recent retrospective study, reviewed 14 patients surgically treated for a displaced medial clavicle fracture between 2010 and 2017. Locking Compression Plates were used. The mean QuickDASH score was 0.81 and the mean SSV was 96. As complications, one patient had an early revision operation and developed an infection after 1.5 years. Eight patients had their implants removed. The authors concluded that operative treatment of displaced medial clavicle fractures with well-fitting 'small fragment' locking plates provides an excellent long-term functional outcome [13].

Sidhu, et al. investigated 27 patients with a median age of 37 years undergoing operative fixation of a medial clavicle fracture, 7 physeal injuries, and 20 adult injuries. The fracture was fixed with plate and screws in 19 cases and with transosseous sutures in 8 cases. The median DASH score at 12 months was 0.4 with a union rate of $100 \%$ at 12 months. All patients had full shoulder range of motion at final follow-up and were able to return to preinjury occupational activities. These authors concluded that early intervention could minimize the risk of painful nonunion [14].

In this case, the implanted osteosynthesis material was able to promote a safe fixation with callus formation and bone remodeling. Such in the previous studies presented, the patient had a good and full recovery with no registered complications.

\section{Conclusion}

Considering the rarity of medial clavicle fractures 
associated with significant displacement and the few reported cases in the literature concerning their surgical treatment, the authors collected this case in order to report this rare and complex injury, and how they decided to manage it. Thus, the treatment chosen seems to give a good functional and radiographic recovery.

\section{References}

1. Postacchini F, Gumina S, De Santis P, Albo F (2002) Epidemiology of clavicle fractures. J Shoulder Elbow Surg 11: $452-456$

2. Nowak J, Mallmin H, Larsson S (2000) The aetiology and epidemiology of clavicular fractures. A prospective study during a two-year period in Uppsala, Sweden. Injury 31: 353-358.

3. Low AK, Duckworth DG, Bokor DJ (2008) Operative outcome of displaced medial-end clavicle fractures in adults. J Shoulder Elbow Surg 17: 751-754.

4. McKenna M (2009) Plating of a periarticular medial clavicle fracture. Orthopedics 32: 366.

5. Van Laarhoven JJ, Hietbrink F, Ferree S, Gunning AC, Houwert RM, et al. (2016) Associated thoracic injury in patients with a clavicle fracture: A retrospective analysis of 1461 polytrauma patients. Eur J Trauma Emerg Surg.

6. Kälicke T, Andereya S, WesthoV J, Gekle Ch (2003) Anterior sternoclavicular dislocation caused by indirect compression trauma. Eur J Trauma 5: 327-329.
7. O'Connor PA, Nölke L, O'Donnell A, Maha Lingham K (2003) Retrosternal dislocation of the clavicle associated with a traumatic pneumothorax. Interact Cardiovasc Thorac Surg 2: 9-11.

8. Khalid N, Elbeshbeshy A, Alsaleh KA, Al-Ahaideb A (2013) Anterior sternoclavicular dislocation associated with clavicular fracture: A case report and review of the literature. Eur J Orthop Surg Traumatol 23: S179-S182.

9. Oe K, Gaul L, Hierholzer C, Woltmann A, Miwa M, et al. (2012) Operative management of periarticular medial clavicle fractures-report of 10 cases. J Trauma Acute Care Surg 72: E1-E7.

10. Sewell MD, Al-Hadithy N, Le Leu A, Lambert SM (2013) Instability of the sternoclavicular joint: Current concepts in classification, treatment and outcomes. Bone Joint $\mathrm{J}$ 95: 721-731.

11. Nowak J, Holgersson M, Larsson S (2005) Sequelae from clavicular fractures are common: A prospective study of 222 patients. Acta Orthop 76: 496-502.

12. Throckmorton T, Kuhn JE (2007) Fractures of the medial end of the clavicle. J Shoulder Elbow Surg 16: 49-54.

13. Frima H, Houwert RM, Sommer C (2018) Displaced medial clavicle fractures: Operative treatment with locking compression plate fixation. Eur J Trauma Emerg Surg.

14. Sidhu VS, Hermans D, Duckworth DG (2015) The operative outcomes of displaced medial-end clavicle fractures. J Shoulder Elbow Surg 24: 1728-1734. 